\title{
The Australasian Society for Infectious Diseases guidelines for the diagnosis, management and prevention of infections in recently arrived refugees: an abridged outline
}

\author{
Ronan J Murray, Joshua S Davis and David P Burgner on behalf of the \\ Australasian Society for Infectious Diseases Refugee Health Guidelines Writing Group*
}

A ustralia and New Zealand have obligations under the 1951 United Nations Convention relating to the Status of Refugees and the 1967 Protocol Relating to the Status of Refugees to assist in the relocation of individuals who are unable to remain in their country of origin. Currently, about 13000 refugees migrate to Australia each year, ${ }^{1}$ with priority regions for individuals needing resettlement including southern and South-East Asia, the Middle East and sub-Saharan Africa. ${ }^{1}$

The Australian Government requires that refugees being considered for migration to Australia undergo a health assessment before being issued with a visa. This assessment includes screening for HIV infection in those aged 15 years or over, and for active tuberculosis infection (including a chest radiograph) in those older than 11 years. ${ }^{2}$ An additional "fitness-to-fly" pre-departure assessment is usually performed shortly before travelling to Australia. Testing for and treatment of malaria and empiric treatment for helminth infection have recently been added to the fitness-to-fly assessment for many sub-Saharan African refugees. However, as refugees bear a disproportionate burden from other acute and chronic infectious diseases that may be undiagnosed or untreated at the time of arrival in Australia, ${ }^{3-5}$ timely post-arrival screening for infectious diseases and other common conditions in all refugees is essential, to ensure not only the health of each refugee but also the public health of the broader Australian community.

In recent years, migrant and refugee health service providers have noted an increase in the number and variety of infectious diseases in newly arrived refugees, particularly in those from subSaharan Africa. ${ }^{3-5}$ These infections are often unfamiliar to local

\section{Key general recommendations}

- All refugees should be offered a comprehensive health assessment, ideally within 1 month of arrival in Australia. This should include:

$>$ screening for and treatment of tuberculosis, malaria, bloodborne viral infections, schistosomiasis, helminth infection, and sexually transmitted infections;

$>$ testing for and treatment of other infections (eg, Helicobacter pylori) as indicated by clinical assessment; and

$>$ assessment of immunisation status, and catch-up immunisations where appropriate.

- The assessment can be undertaken by a general practitioner or within a multidisciplinary refugee health clinic.

- An appropriate interpreter should be used when required.

- The initial assessment should take place over at least two visits: the first for initial assessment and investigation and the second for review of results and treatment or referral.

- Psychological, dental, nutritional, reproductive and developmental health issues (which are beyond the scope of these guidelines) should also be addressed at the post-arrival health assessment.

\section{ABSTRACT}

- About 13000 refugees are currently accepted for migration into Australia each year, many of whom have spent protracted periods living in extremely disadvantaged circumstances. As a result, medical practitioners are increasingly managing recently arrived refugees with acute and chronic infectious diseases.

- The Australasian Society for Infectious Diseases has formulated guidelines for the diagnosis, management and prevention of infection in newly arrived refugees. This article is an abridged version of the guidelines, which are available in full at <http://www. asid.net.au>.

- All refugees should be offered a comprehensive health assessment, ideally within 1 month of arrival in Australia, that includes screening for and treatment of tuberculosis, malaria, blood-borne viral infections, schistosomiasis, helminth infection, sexually transmitted infections, and other infections (eg, Helicobacter pylori) as indicated by clinical assessment; and assessment of immunisation status, and catch-up immunisations where appropriate.

- The assessment can be undertaken by a general practitioner or within a multidisciplinary refugee health clinic, with use of an appropriate interpreter when required. The initial assessment should take place over at least two visits: the first for initial assessment and investigation and the second for review of results and treatment or referral.

MJA 2009; 190: 421-425

medical practitioners, as many are not endemic to Australia. This has led to a degree of uncertainty and concern among refugees, health care providers and the wider community. How should we screen for infection in refugees? Which tests should be performed? What should we do with the results? What about catch-up immunisations? The states and territories have developed their

* Other members of the Writing Group were Meredith Hansen-Knarhoi, Vick Krause, Beverley-Ann Biggs, Christopher Lemoh, Jill Benson, Sarah Cherian, Jim Buttery and Georgia Paxton.

A working group determined a list of priority conditions and issues to be included in the guidelines, and 11 members were assigned section-writing responsibilities. First drafts of each section were internally reviewed and revised; second drafts were externally reviewed by seven experts in relevant fields, returned to section authors and compiled into a single document, with a recommendation table. The draft was circulated to stakeholders (the Communicable Diseases Network Australia [CDNA] and the Australasian Chapter of Sexual Health Medicine [AChSHM] of the Royal Australasian College of Physicians) and to all members of the Australasian Society for Infectious Diseases (ASID) for review. Comments from stakeholders were returned to authors, and the final document was prepared and endorsed by ASID, CDNA, the National Tuberculosis Advisory Committee and AChSHM. 


\section{REFUGEE HEALTH}

\section{Summary of recommendations for the diagnosis, treatment and prevention of common infections in recently arrived refugees*}

\section{Tuberculosis}

Recommended screening test: Mantoux test or interferon- $\gamma$ release assay

If positive:

- Refer to local tuberculosis (TB) service for exclusion of active TB infection and/or consideration of treatment of latent TB infection (Level I)

\section{Malaria}

Recommended screening test: malaria thick and thin blood films and Plasmodium falciparum antigen test

If positive:

- Discuss urgently with local infectious disease service and refer for treatment

\section{Blood-borne viral infections}

Recommended screening test: HIV serology

If positive:

- Refer to local HIV service

- Advise of transmission risk

- Ensure TB screening has been performed

- Ensure screening for other blood-borne viruses has been performed

Recommended screening test: hepatitis $B(H B)$ serology (sAg/sAb/cAb) If HBsAg positive:

- Request HBeAg and liver function tests (LFTs)

- If HBeAg positive OR LFT results abnormal, refer to local viral hepatitis management service

- Advise of transmission risk

- Immunise all non-immune household contacts

Recommended screening test: hepatitis $C$ serology If positive:

- Request LFTs and hepatitis C virus (HCV) RNA (qualitative)

- Advise of transmission risk

- If LFT results abnormal AND/OR HCV RNA detected, refer to local viral hepatitis management service

\section{Sexually transmitted infections}

Recommended screening test: syphilis serology

If positive:

- Review history, including previous treatment

- Treat as per local guidelines

- Discuss cases of all children with positive serological test results for syphilis with paediatric infectious disease service

Recommended screening test: nucleic acid detection test for Chlamydia trachomatis and Neisseria gonorrhoeae

All adults and others who are sexually active or may have been sexually assaulted should be screened for chlamydia and gonorrhoea infection on first-void urine
Helminth infection

Recommended screening test: Strongyloides serology

If positive:

- Ivermectin $200 \mu \mathrm{g} / \mathrm{kg}$ orally as a single dose, repeated 14 days after first dose (Level II)

- If patient is aged < 5 years, do not give ivermectin; refer to paediatric infectious disease service

Recommended screening test: full blood count (FBC) and/or faeces microscopy

If faeces readily obtainable OR symptoms present, faeces microscopy should be followed by directed treatment

If faeces not readily obtainable AND patient is asymptomatic:

- If no documented pre-departure albendazole therapy:

$>$ Empiric single-dose albendazole (patient $\leqslant 10$ kg, 200 mg; $>10 \mathrm{~kg}, 400 \mathrm{mg}$ ) (Level I)

$>$ Repeat FBC in 8 weeks; if eosinophilia still present, investigate further or specialist referral

- If documented pre-departure albendazole therapy:

$>$ No eosinophilia: no further treatment or follow-up

> Eosinophilia: repeat FBC in 8 weeks - if eosinophilia still present, investigate further or specialist referral

\section{Schistosomiasis}

Recommended screening test: schistosomiasis serology

If positive:

- Praziquantel $20 \mathrm{mg} / \mathrm{kg}$, two doses 4 hours apart (Level I)

(30 mg/kg if patient is from South-East Asia) (Level II)

- Obtain stool and urine for ova examination

- Refer patients with chronic liver disease (including viral hepatitis), symptoms or signs for further assessment

Other infections that may be detected at the health assessment - indications for testing

Helicobacter pylori infection

Adults with suspected peptic ulcer disease (based on symptoms):

- Non-invasive tests for $\mathrm{H}$. pylori infection (eg, stool antigen test or urea breath test)

- If positive, treat as per current guidelines

Children with anorexia, poor weight gain or failure to thrive should be referred to a paediatric refugee health service for assessment

Uncommon infections (eg, filariasis, tungiasis)

Discussion with or referral to an adult or paediatric infectious disease specialist is recommended

\section{Immunisation}

Catch-up immunisations for all ages in accordance with the Australian Standard Immunisation Schedule, unless there is written evidence of adequate immunisation; serological tests to detect existing immunity are not recommended 
own responses to these challenges, with approaches varying from no or limited screening to comprehensive screening for both common and rarer infections. ${ }^{3-7}$

In late 2005, the Communicable Diseases Network Australia (CDNA) asked the Australasian Society for Infectious Diseases (ASID) to formulate guidelines for the screening, treatment and prevention of infections in recently arrived sub-Saharan African refugees, with the aim of providing practical assistance to general practitioners and others who provide health care services to this population (see footnote on page 421 for details of the guideline development process). Here, we summarise the major recommendations of these guidelines, which are available in full on the ASID website at <http://www.asid.net.au>. ${ }^{8}$ Treatment recommendations have been graded according to National Health and Medical Research Council (NHMRC) levels of evidence. ${ }^{9}$

Although the guidelines were originally written for refugees originating from sub-Saharan Africa, many of the recommendations will apply to refugees from other regions. Each section of the guidelines includes a discussion of issues specific to refugee children. The guidelines have been endorsed by the CDNA, the National Tuberculosis Advisory Committee, and the Australasian Chapter of Sexual Health Medicine of the Royal Australasian College of Physicians.

The key general recommendations of the guidelines are shown in Box 1, and a summary of recommendations for testing for and treatment of common infections in refugees is provided in Box 2. More detailed recommendations for some of the most commonly encountered infections are shown in Box 3 (tuberculosis, malaria and schistosomiasis) and Box 4 (blood-borne viral infections [HIV, hepatitis B and hepatitis C] and sexually transmitted infections). Wherever possible, treatment recommendations are based on published evidence; however, there is a clear need for further research to develop a broader evidence base to underpin the management of the complex health needs of refugees.

Newly arrived refugees have been through significant turmoil and upheaval. Therefore, post-arrival health assessments must be sensitive to their feelings of cultural disorientation, vulnerability and sometimes fear and mistrust of authorities. We have recommended a screening approach that can be performed by a single venepuncture (see Box 2), avoiding multiple blood tests and routine collection of other specimens wherever possible. We believe that the guidelines strike a balance between identifying important and treatable infections and overinvestigation.

These guidelines focus on infectious diseases. However, assessment for infectious diseases is only one component of a comprehensive overall assessment that should also address psychological health, nutritional status, sexual and reproductive health, dental health, chronic disease, cancer screening, and childhood growth and development. We refer readers to other recent Australian publications for advice regarding these important issues, ${ }^{6,7,26,27}$ which are beyond the scope of these guidelines.

It is important to note that unless the granting of an individual's visa was contingent on a specific health undertaking, assessment and screening is offered on a voluntary basis and following informed consent. Refugee health assessments should always be undertaken with an interpreter, either in person or by telephone. The interpreter should not be related to the patient, and the need for confidentiality should be emphasised. The Australian Government provides the Translating and Interpreting Service (TIS) for people who do not speak English and for those who need to

\section{Detailed recommendations for tuberculosis, malaria} and schistosomiasis*

\section{Tuberculosis (TB)}

With the exception of those with documented past TB disease, all newly arrived refugees, including children, should be assessed for latent TB infection (LTBI), with the following plan:

- Testing is performed with the intention to treat. ${ }^{10}$

- Either a Mantoux test or a blood-based interferon- $\gamma$ release assay (IGRA) may be used for screening. ${ }^{11,12}$

- A Mantoux test result $\geqslant 10 \mathrm{~mm}$ in adults and children $\geqslant 5$ years of age and $\geqslant 5 \mathrm{~mm}$ in those younger than 5 years or those who are HIV-infected is considered positive.

- Refer those with a positive Mantoux test or blood-based IGRA result to local TB service for exclusion of active TB infection and consideration of treatment of LTBI. ${ }^{13}$ (Level I)

- Refugees known to be HIV-infected should have a two-step Mantoux test. If the second test result remains $<5 \mathrm{~mm}$, specialist advice should be sought from TB/HIV services.

- TB (active disease or latent infection) should be managed by clinicians experienced in doing so as part of a centralised, coordinated TB service.

\section{Malaria}

- All refugees, including those who have had documented testing and/or treatment for malaria at the time of pre-departure assessment, ${ }^{14}$ should be tested for malaria after arrival in Australia (except those who have never resided in or travelled through a region where malaria occurs).

- Testing should be performed both by thick and thin blood films AND an antigen-based rapid detection test. ${ }^{15}$

- All cases of malaria should be treated by or in consultation with a specialist infectious disease service.

- Falciparum malaria in adults resettled in malaria non-receptive areas of Australia may be treated in the outpatient setting if the following criteria are satisfied: asymptomatic or minimally symptomatic, not pregnant, and no indicators of severe malaria (altered consciousness, jaundice, oliguria, severe anaemia or hypoglycaemia, parasite count $>100000 / \mu \mathrm{L}$ or $>2 \%$, or patient is vomiting or acidotic). ${ }^{16}$ (Level IV)

- Cases of malaria in children should be urgently discussed with a paediatric infectious disease service. ${ }^{17}$

\section{Schistosomiasis}

- Serological tests for schistosomiasis should be performed for all recently arrived African and South-East Asian refugees. ${ }^{18,19}$

- Those with negative results of serological tests do not require further investigation.

- Those with positive results of serological tests should be treated presumptively:

> Praziquantel $40 \mathrm{mg} / \mathrm{kg}$ in two doses of $20 \mathrm{mg} / \mathrm{kg}$, 4 hours apart for refugees from Africa. ${ }^{20-22}$ (Level I)

$>$ Praziquantel $60 \mathrm{mg} / \mathrm{kg}$ in two doses of $30 \mathrm{mg} / \mathrm{kg}$, 4 hours apart for refugees from South-East Asia. ${ }^{23}$ (Level II)

- Those with positive results of serological tests should also have faeces and urine examined for schistosoma ova to determine if further follow-up is required (refer to full guidelines ${ }^{8}$ for details).

* Treatment recommendations graded according to National Health and Medical Research Council (NHMRC) levels of evidence. ${ }^{9}$ 


\section{Detailed recommendations for blood-borne viral and sexually transmitted infections}

\section{Blood-borne viral infections (HIV, hepatitis B and hepatitis C)}

- Screening for HIV, hepatitis B and hepatitis $C$ infection should be offered to all refugees.

- All screening tests should be performed with the knowledge and informed consent of the individual or his/her legal guardian. Discussion of these screening tests and communication of results must be carried out with utmost respect for the privacy of the individual. Practitioners should explicitly state their obligations to protect patient confidentiality, but also their obligation to notify the relevant authorities of the diagnosis.

- Practitioners should explain that the results of screening tests do not have adverse implications for the refugees' status as Australian residents.

- Testing the mother for HIV infection should not be used in lieu of direct testing of the child.

- Refugees with positive results of serological tests for HIV should be advised of the transmission risk (including vertical transmission if pregnant) and referred urgently to the local HIV management service.

- Those with positive results of serological tests for HCV should also have HCV polymerase chain reaction (PCR) tests and liver function tests (LFTs) performed. Patients with a positive PCR test result OR abnormal LFT results should be referred to the local viral hepatitis management service.

- Those with positive HBsAg test results should have $\mathrm{HBeAg}$ and eAb tested, as well as LFTs. Patients with a positive eAg OR abnormal LFT results should be referred to the local viral hepatitis management service. Non-HBV-immune household contacts of an HBV-infected individual should be immunised against HBV.

\section{Sexually transmitted infections}

- All refugees (including children) should have serological testing for treponemal infection (syphilis), with an intention to treat all those with positive results in whom there is no documented history of prior treatment. ${ }^{24}$

- All adult refugees should be tested for Chlamydia trachomatis and Neisseria gonorrhoeae genital infection using a nucleic acid detection (NAD) test on first-void urine. This should also be performed in any youth who is sexually active or who may have been sexually assaulted. ${ }^{25}$

- All adult female refugees should be offered a "well women's check", including screening for cervical cancer. A cervical swab should be collected at the same time as an NAD test to detect Chlamydia trachomatis and Neisseria gonorrhoeae infection. ${ }^{25}$

- Investigation for other sexually transmitted infections should be performed according to history, symptoms and signs elicited at the initial assessment.

$\mathrm{HCV}=$ hepatitis $C$ virus. $\mathrm{HBV}=$ hepatitis $B$ virus. $H B s A g=$ hepatitis $B$ virus surface antigen. $\mathrm{HBeAg}=$ hepatitis $B$ virus e antigen. $\mathrm{eAb}=$ anti-hepatitis $B$ virus e antigen antibody.

communicate with them. This service is available free of charge to practitioners who provide a Medicare service within a private practice to permanent residents or citizens who do not speak English. The service is also available within hospitals or government-funded clinics, but must be paid for in these settings. The TIS Doctors Priority Line (tel: 1300131 450) should be used to access this service.

\section{Competing interests}

None identified.

\section{Author details}

Ronan J Murray, MB BS, FRACP, FRCPA, Infectious Diseases Physician and Clinical Microbiologist ${ }^{1}$

Joshua S Davis, MB BS, DTM\&H, FRACP, Infectious Diseases Physician, ${ }^{2}$ and NHMRC Scholar ${ }^{3}$

David P Burgner, MB ChB, FRACP, PhD, Paediatric Infectious Diseases and Refugee Child Health Physician, ${ }^{4}$ and Associate Professor in Paediatrics ${ }^{5}$

1 Royal Perth Hospital and PathWest Laboratory Medicine WA, Perth, WA.

2 Royal Darwin Hospital, Darwin, NT.

3 Menzies School of Health Research, Darwin, NT.

4 Child and Adolescent Health Service, Western Australian Department of Health, Perth, WA.

5 School of Paediatrics and Child Health, University of Western

Australia, Perth, WA.

Correspondence: ronan.murray@health.wa.gov.au

\section{References}

1 Australian Government Department of Immigration and Citizenship. Fact Sheet 60 - Australia's Refugee and Humanitarian Program. Canberra: Department of Immigration and Citizenship, 2007. http://www.immi. gov.au/media/fact-sheets/60refugee.htm (accessed Aug 2008).

2 Australian Government Department of Immigration and Citizenship. Health requirement for permanent entry to Australia. Form 1071i. Canberra: Department of Immigration and Citizenship, 2007. http:// www.immi.gov.au/allforms/pdf/1071i.pdf (accessed Aug 2008).

3 Martin JA, Mak DB. Changing faces: a review of infectious disease screening of refugees by the Migrant Health Unit, Western Australia in 2003 and 2004. Med J Aust 2006; 185: 607-610.

4 Tiong ACD, Patel MS, Gardiner J, et al. Health issues in newly arrived African refugees attending general practice clinics in Melbourne. Med J Aust 2006; 185: 602-606.

5 Cooley L, Nott L, Williams M, McGregor A. Prevalence of selected infectious diseases in an African refugee population [abstract]. Intern Med J 2004; 34 (9-10 Suppl): A75.

6 Smith MM. Refugees in Australia: changing faces, changing needs [editorial]. Med J Aust 2006; 185: 587-588.

7 Benson J, Smith MM. Early health assessment of refugees. Aust Fam Physician 2007; 36: 41-43.

8 Murray R, Davis J, Krause V, et al; Australasian Society for Infectious Diseases Refugee Health Guidelines Writing Group. Diagnosis, management and prevention of infections in recently arrived refugees. Sydney: Dreamweaver Publishing, 2009. http://www.asid.net.au/guidelinesandpublications/ (accessed Mar 2009).

9 National Health and Medical Research Council. A guide to the development, implementation and evaluation of clinical practice guidelines. Canberra: NHMRC, 1999.

10 Porco TC, Lewis B, Marseille E, et al. Cost-effectiveness of tuberculosis evaluation and treatment of newly-arrived immigrants. BMC Public Health 2006; 6: 157.

11 Pai M, Riley LW, Colford JM Jr. Interferon-gamma assays in the immunodiagnosis of tuberculosis: a systematic review. Lancet Infect Dis 2004; 4: 761-776.

12 Mazurek GH, Jereb J, Lobue P, et al. Guidelines for using the QuantiFERON-TB Gold test for detecting Mycobacterium tuberculosis infection, United States. MMWR Recomm Rep 2005; 54 (RR-15): 49-55.

13 Smieja MJ, Marchetti CA, Cook DJ, Smaill FM. Isoniazid for preventing tuberculosis in non-HIV infected persons. Cochrane Database Syst Rev 2000; (2): CD001363. doi: 10.1002/14651858.CD001363.

14 Cherian S, Fagan JM, Thambiran A, et al. Severe Plasmodium falciparum malaria in refugee children despite reported predeparture antimalarial treatment [letter]. Med J Aust 2006; 185: 611.

15 Farcas GA, Zhong KJ, Lovegrove FE, et al. Evaluation of the Binax NOW ICT test versus polymerase chain reaction and microscopy for the 


\section{REFUGEE HEALTH}

detection of malaria in returned travelers. Am J Trop Med Hyg 2003; 69: 589-592.

16 Chih DT, Heath $\mathrm{CH}$, Murray RJ. Outpatient treatment of malaria in recently arrived African migrants. Med J Aust 2006; 185: 598-601.

17 Cherian S, Burgner D. Selective ambulatory management of Plasmodium falciparum malaria in paediatric refugees. Arch Dis Child 2007; 92: 983986.

18 Tsang VC, Wilkins PP. Immunodiagnosis of schistosomiasis. Immunol Invest 1997; 26: 175-188.

19 Davis JS, Webber MT. A prospective audit of infectious diseases in 222 newly arrived African refugees [abstract]. J Paediatr Child Health 2006; 42: A13.

20 Ross AGP, Bartley PB, Sleigh AC, et al. Schistosomiasis. N Engl J Med 2002; 346: 1212-1220.

21 Danso-Appiah A, Utzinger J, Liu J, Olliaro P. Drugs for treating urinary schistosomiasis. Cochrane Database Syst Rev 2007; (4): CD000053. doi: 10.1002/14651858.CD000053.pub2.

22 Saconato H, Atallah A. Interventions for treating schistosomiasis mansoni. Cochrane Database Syst Rev 1999; (1): CD000528. doi: 10.1002/ 14651858.CD000528.
23 Santos AT, Blas BL, Nosenas JS, et al. Preliminary clinical trials with praziquantel in Schistosoma japonicum infections in the Philippines. Bull World Health Organ 1979; 57: 793-799.

24 Centers for Disease Control and Prevention. Notice to readers: recommendation regarding screening of refugee children for treponemal infection. MMWR Morb Mortal Wkly Rep 2005; 54: 933-934.

25 Australasian College of Sexual Health Physicians. Clinical guidelines for the management of sexually transmissible infections among priority populations. 2004. http://www.wsahs.nsw.gov.au/services/sexualhealth/ documents/STI_Management_Priority_Populations_000.pdf (accessed Mar 2009).

26 The Victorian Foundation for Survivors of Torture. Caring for refugee patients in general practice: a desk-top guide. 3rd ed. 2007. http:// www.foundationhouse.org.au/resources/publications_and_ resources.htm (accessed Aug 2008).

27 Davidson N, Skull S, Chaney G, et al. Comprehensive health assessment for newly arrived refugee children in Australia. J Paediatr Child Health 2004; 40: 562-568.

(Received 15 Jun 2008, accepted 3 Sep 2008) 